\title{
A Cross-Cultural Perspective on Challenges Facing Comparative Cancer Survivorship Research
}

\author{
Astri Syse ${ }^{1,2}$ and Berta Geller ${ }^{3}$ \\ ${ }^{1}$ Cancer Registry of Norway, P.O. Box 5313 Majorstua, 0304 Oslo, Norway \\ ${ }^{2}$ Dartmouth Medical School, Norris Cotton Cancer Center, Lebanon, NH 03755, USA \\ ${ }^{3}$ Office of Health Promotion Research, University of Vermont, 1 South Prospect Street UHC, Burlington, VT 05401, USA
}

Correspondence should be addressed to Astri Syse, astri.syse@kreftregisteret.no

Received 11 May 2011; Revised 9 August 2011; Accepted 16 August 2011

Academic Editor: P. Vineis

Copyright (C) 2011 A. Syse and B. Geller. This is an open access article distributed under the Creative Commons Attribution License, which permits unrestricted use, distribution, and reproduction in any medium, provided the original work is properly cited.

Cancer survivorship research includes the study of physical, psychosocial, and economic consequences of cancer diagnosis and treatment among pediatric and adult cancer survivors. Historically, the majority of cancer survivorship studies were from the United States, but survivorship issues are increasingly being addressed in other developed countries. Cross-cultural studies remain, however, scarce. The degree to which knowledge attained may or may not be transferred across cultures, countries, or regions is not known. Some important challenges for comparative research are therefore discussed in a cross-cultural perspective. Several substantive and methodological challenges that complicate the execution of cross-cultural cancer survivorship research are presented with examples and discussed to facilitate comparative research efforts in the establishment of new survivorship cohorts and in the planning and implementation of survivorship studies. Comparative research is one key to understanding the nature of cancer survivorship, distinguishing modifiable from nonmodifiable factors at individual, hospital, societal, and system levels and may thus guide appropriate interventions. Lastly, suggested future courses of action within the field of comparative cancer survivorship research are provided.

\section{Introduction}

The emerging discipline of cancer survivorship research includes the study of the consequences of cancer diagnosis and its treatment among children and adults who have experienced cancer [1]. While the United States (USA) has conducted the majority of research on adult cancer survivorship [2-6], the United Kingdom and Canada have conducted large-scale cohort studies of childhood cancer survivorship $[7,8]$. As more countries are beginning to address issues related to adult survivorship [9-20], it will be important to consider how transferrable findings will be across cultures and nations. Identifying generalizable and nongeneralizable research among cultures will hasten what we learn about cancer survivorship. This essay presents an overview of cancer survivorship research and its challenges and discusses in detail three examples of pronounced crosscultural challenges currently facing the field. Finally, future courses of action within the field of comparative cancer survivorship research are suggested.

\section{Cancer Survivorship Research}

According to the US Institute of Medicine, cancer survivorship research encompasses the physical, psychosocial, and economic consequences of cancer diagnosis and its treatment among both pediatric and adult survivors of cancer [1]. It seeks to "identify, examine, prevent, and control adverse cancer- and treatment-related outcomes" and to "provide a knowledge base regarding optimal followup care and surveillance of cancer survivors" [1]. Lastly, cancer survivorship research aspires to "optimize health after cancer treatment", seeking to promote healthy lifestyles and behaviors. A cancer survivor is a person diagnosed with cancer, current or past, who is still living [1]. Originally, the medical community limited the term to denote persons 
who remained alive and disease-free for a minimum of five years, thus reflecting "cure." Today's cancer survivors may be disease-free or not and may experience no, minor or major short- or long-term effects such as pain, sexual dysfunction, second cancers, or poor quality of life from cancer illness and/or treatment. Cancer survivorship denotes the phase following primary treatment until end-of-life care or death. Family members and caregivers may also be impacted by the survivorship experience and are therefore included in the survivorship definition, as are their processes related to coping and/or grief $[1,5]$.

\section{Scope of Today's Research}

Cancer is one of the most common serious illnesses in the developed world today, and between one in three and one in two people can be expected to be diagnosed with cancer during their lifetime [21]. Cancer incidence increases with age and is consequently rising in societies where life expectancy is increasing. Concurrently, the number of cancer cases is increasing due to cancer screening and improved diagnostic tests, population growth, and changes in underlying risk factors, such as environmental exposures, obesity, and physical inactivity $[1,5,21]$. At the same time, prognosis is improving for most cancers, and the overall five-year relative survival after cancer is above $60 \%$ in most developed countries due to early detection and improved treatment $[5,21]$. Europe has the highest number of cancer survivors-close to 14 million, while there are about 12 million in Canada and the USA, about 8 million in SouthEastern Asia, but only about 1 million in Oceania [21, 22]. This results in an estimated population of more than 35 million cancer survivors in the developed world alone, corresponding to a population prevalence of about $3.5 \%$. The absolute number of cancer survivors is predicted to increase nearly threefold over the next few decades, while the number of cancer deaths is expected to double [21]. This dramatic increase will have profound implications for the individuals directly affected by cancer and for societies who attend to their needs.

Cancer incidence and cause-of-death registration is essential for identifying cancer survivors and for understanding the complex interplay. In many parts of the developing world (e.g., most of Africa) resources for cancer registration are not available [21]. Less than $20 \%$ of the world's population is covered by cancer registries and around $30 \%$ by mortality registration [22], and the total number of cancer survivors in the world is thus unknown. Research on cancer survivorship is currently primarily undertaken in countries with adequate cancer surveillance and sufficient resources available to care for persons after a diagnosis has been made [23].

Cancer survivorship research is currently conducted in many ways, reflecting the complexities of the subject matter. Like in other areas of health research data, data may be collected prospectively [12] or retrospectively [24], and both objective [25] and subjective [10,24] measures of exposures and outcomes are relevant. The data sources span from administrative records collected for other purposes such as billing, to hospital, state and national cancer registry information $[11,12,26]$, clinical trials $[27,28]$, as well as information from questionnaires, interviews, or observation $[12,18]$. In certain instances, more precise answers and further tests may be required from subjects, including biological samples (e.g., blood, urine, saliva) or physical stress tests or tests to measure cognitive performance $[29,30]$, as well as different imaging techniques [31]. The majority of studies to date have included only cancer survivors, but some questions are impossible to answer without noncancer comparison groups $[10,19,25]$. Essential components of cancer survivorship research are the study of short-term, long-term, and late organic and somatic effects following cancer, treatment, and cancer control interventions. The most commonly reported long- and short-term physical complaints after cancer are pain, digestive problems, neuropathies in the extremities, cardiovascular problems, immune deficiency, anemia, lymphedema, impaired vision due to cataracts, impaired mobility, and fatigue $[10,32]$. Research has focused on adverse effects of cancer treatment on cognitive functioning such as memory, attention, and concentration [33]. Cancer has also been reported to affect psychological health by increasing depression and anxiety [34]. A relatively new avenue of research focuses on the economic and legal issues, particularly the economic sequelae of cancer and cancer treatment on individuals, families, and societies $[15,35,36]$. Cancer survivorship research also includes the promotion of healthy lifestyles and behaviors studying the effects of interventions to reduce smoking, increase physical fitness, and reduce weight [37]. Benefits of having survived cancer have been identified through research, that is, posttraumatic growth [24, 38]. Research on the role of families on survivors' health-related and psychosocial outcomes as well as cancer survivorship's impact on health and wellbeing on family units, members, and caregivers is also emerging $[12,39]$. Recurrence and second primary cancers are of particular concern [26]. Lastly, issues related to followup care, access, and health care delivery are in the forefront, with concerns about disparities related to poverty-related or other sociodemographic patient characteristics, with a focus on underserved populations [40-42].

\section{Current Challenges}

In cancer survivorship research, diverse information must be collected and assessed over extended time periods and from multiple sources, including the patient, family members, caregivers, clinics, health care systems, and policy. This is both practically and methodologically difficult and costly [6]. As is the case for many emerging research fields, the challenges facing the field of cancer survivorship research are both substantive and methodological.

One important substantive challenge is to decide which research questions to ask and what hypotheses to test in this relatively new field. Economic, legal, social, physical, spiritual, emotional aspects, among others, have been identified as content areas, but the relative importance placed on each of these may vary between cultures because cultures 


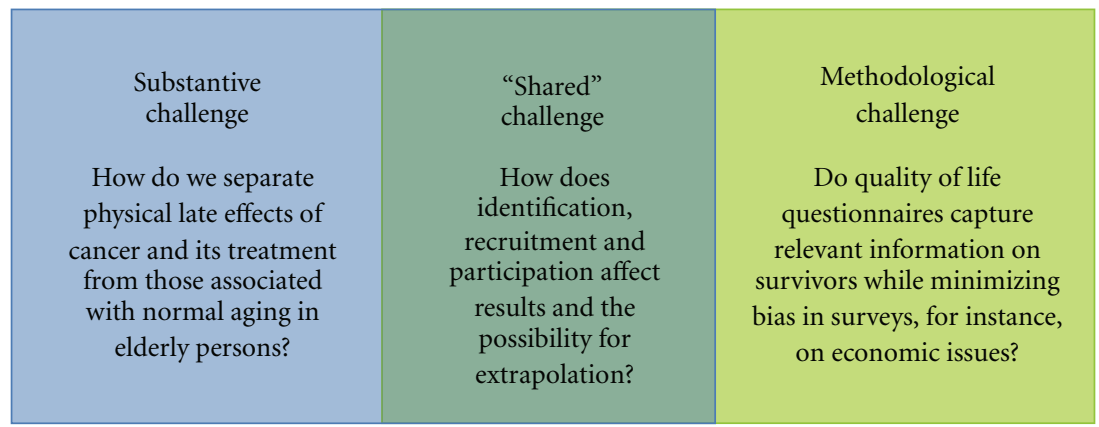

FIgURE 1: An example of a substantive, a methodological, and a "shared" challenge.

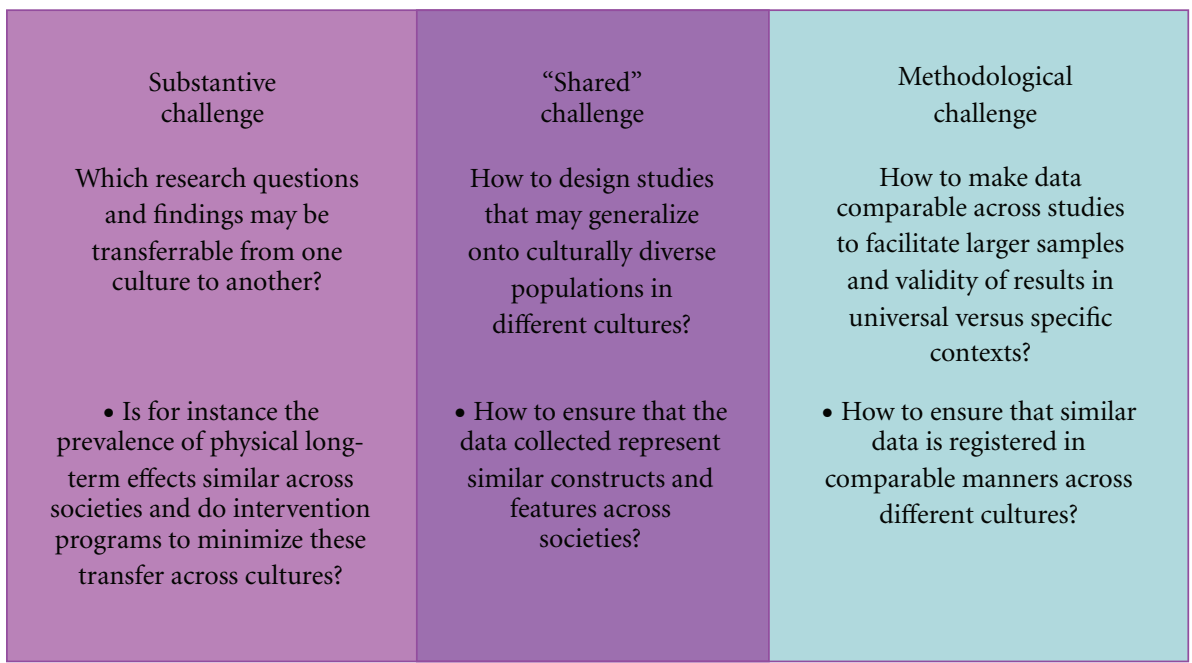

FIGURE 2: An illustration of possible groupings of cross-cultural challenges.

view and value illness, treatments, and caregiving differently $[20,42,43]$. Are there common beliefs and attitudes among different cultures that can be studied to benefit multiple societies? What research questions and findings transfer across societies? Which questions must be answered within subcultures due to large contrasts between cultures or societies? The answers to these questions impact on how limited research funding can be distributed to optimize the knowledge gained and thus augment cancer survivorship care, cross-culturally.

Different methodological challenges complicate cancer survivorship research. As researchers we must learn how to best measure the effects of surviving cancer and how to increase overall and subgroup research participation and followup. To be efficient we need to use existing data sources while identifying new sources, and apply existing research methods, while designing and testing new methods. Assessing effects from multiple sources and over time may call for new and advanced research methods [44]. Researchers with expertise in research design, measurement, and analyses must be included. Finally, when considering design and methods we must keep in the forefront the need to review costs and benefits as they relate to the generalizability of the findings to different populations.
The application of research findings to individuals and subgroups across different societies requires an understanding of the substantive concerns of each culture and may thus need to rely on study designs and methods that are acceptable across cultures. These issues are also involved in how one disseminates and uses the results to improve cancer care across different societies. Figure 1 exemplifies challenges of either substantive or methodological character and also provides an example of an area where there is overlap.

The substantive and methodological challenges described above may exist within one culture. As we proceed to describe challenges across cultures, broadly defined to include diverse countries, societies, or groups within these that share distinct features, matters become more complicated. Different cultures may face distinctive substantive challenges. The relative importance of content areas may differ. Also methodological challenges may in part vary across cultures, given different infrastructures, data availability, and legal restrictions, whereas other methodological challenges may have more general relevance. Across cultures, one example of a substantive challenge could be "which research questions and findings may be transferable from one culture to another?", as is shown on the left-hand side in Figure 2. On the right-hand side in this figure, 
an example of a methodological challenge could be "How to make data comparable across studies to facilitate larger samples and validity of results in universal versus specific contexts?" An example of a challenge that cuts across this categorization could be "How to design studies that may generalize to diverse populations in different societies?where more specifically one's concern could be how to ensure that the data collected represent similar constructs and features across societies. Equally important is to recognize what cultural aspects are so unique that they cannot be compared across cultures.

There are three important challenges currently facing cancer survivorship research. Each entails components of both substantive and methodological issues, and we believe that all need to be adequately addressed for cancer survivorship research to move forward. The first challenge is to determine which questions pertain across cultures. The second challenge concerns how one may assure that what is being measured is what one wants to measure across cultures. It also encompasses how to extrapolate research findings. Lastly, the challenge of securing nondifferential participation cross-culturally, initially, and during followup will be addressed.

\subsection{Challenge I: Determining Relevant Hypotheses and Out-} comes within and across Cultures. Certain research questions are important to ask cross-culturally, whereas others may be adequately addressed within (sub)cultures. Research hypotheses, questions, and findings that generalize to other societies and thus translate to larger/diverse populations need not be replicated to the same extent as those that are culturally specific. Distinguishing between unnecessary and important replications is essential to minimize study costs and ensure optimal outcomes for cancer survivors and requires knowledge and insight into the different cultures, systems, and societies. Certain phenomena might need to be studied in different cultures and at various levels to determine whether questions pertain cross-culturally and across levels.

Examples of study questions that might be valid across cultures include for instance the presence of short, long, and late physical effects following cancer and its treatment, as there is little reason to believe that for instance drug side effects, late effects of radiation, or the prevalence of second cancers differ markedly between cultures unless treatment regimens have been substantially different $[10,26,32]$. This was in part the case for pediatric cancer treatment regimens in Europe and the USA a few decades ago [45], and may have contributed to differences in the type and prevalence of somatic late effects across regions. Perceptions and implications of these late effects, may, however, differ vastly between cultures, depending on health and welfare systems, expectations and existing norms [16, 17, 46, 47]. An example of a question that is less likely to be valid across cultures would be one that addresses economic sequelae of cancer. Both the direct costs associated with becoming ill and the indirect costs associated with illness-related work absence are likely to vary as health insurance, followup care, access, and health care delivery differ across cultures [15, 18, 48, 49].
Research on disparities in this area tends to show that the least well off tend to fare the poorest, and poverty or low socioeconomic resources thus becomes one possible target where differential care or followup may be necessary [40-42].

At the community level, one might ask whether results from one hospital are likely to pertain to different hospitals, and similarly for regions and nations. For instance, is research conducted in the USA relevant in European countries and vice versa? Cultural differences in employment behavior, perhaps driven by differences in health and welfare systems, between the USA and Norway are highlighted in this example where we compare employment after cancer. Norway provides universal access to cancer care without costs to individuals, regardless of employment status and a fair percentage stop working after their cancer diagnosis [48]. In the USA, employment rates remain relatively high after cancer, perhaps in part because many who are ineligible for Medicaid or Medicare have their health insurance linked to their employment or the employment of their spouse, frequently with large deductibles and copayments that can forestall treatment and bankrupt families [50]. Further, US breast cancer patients with health insurance tied to their own employment have been found to be more likely to work than those who are covered through their spouse [51]. This would be an irrelevant study question in Norway and other welfare states with universal health care. Likewise may crosscultural and international comparisons facilitate discussions of the relative importance of organizational structures across systems and disclose effects that could be modified within systems. The Aboriginal people in Australia have, for instance, higher cancer mortality than the nonindigenous population, and a study has identified transportation and accommodation problems, travel and health care expenses, removal from family, and lack of appropriate support persons as important factors for treatment decisions and access [42].

\subsection{Challenge II: Determining Relevant Measurements and} Extrapolation of Findings at Different Levels across Cultures. The challenge of securing construct validity and thus contributing to internal validity is present within societies, and even more so across societies. How well we are able to capture the exposures and the outcomes that we want to assess is of outmost importance for our results and conclusions. Physical consequences of cancer and its treatment can be measures both subjectively and objectively, but perceptions of physical consequences or psychosocial issues may only be subjectively reported. The exact relationship between these different types of measures and outcomes has not yet been established within the field of cancer survivorship research. To what degree these measurements capture what they aim to, within or across cultures, remains largely unknown. Because this new research area is in a rush to find results, some studies have performed validation and reported outcomes simultaneously [52]. It is, however, important to distinguish "scale validation research" from studies that attempt to provide information on outcomes as scale development moves forward [53-55], and it is also 
important to clarify the extent to which scales are likely to be valid across cultures.

To further exemplify, the study of physical late effects and psychosocial consequences requires monitoring of large and diverse groups of cancer survivors as the individual adverse events are relatively rare (i.e., second cancers or divorce) and occur at various points in time from diagnosis $[25,26]$. Furthermore, some late effects are relevant only for subgroups of survivors, such as females who received radiation to the chest area for Non-Hodgkin's lymphoma during their reproductive years and later developed breast cancer. In these instances, cross-cultural studies may enable a pooling of study subjects and thus provide sufficient power to answer more questions, as has been done in for instance "The After Breast Cancer Pooling Project", established to examine the role of physical activity, adiposity, dietary factors, supplement use, and quality of life in breast cancer prognosis among US and Chinese survivors [56]. In some instances, pooling might preclude study findings. For instance could pooling of female cancer survivors' employment data from cultures with vastly different female employment patterns yield results that are representative for neither culture.

In addition, there is a lack of evidence-based guidelines for which tests to use and when for surveillance, complicating research measurements. As it is difficult to distinguish effects of "normal" aging, lifestyle behaviors, and comorbidity from that related to cancer, comparable control subjects need to be assessed and followed simultaneously. At present there is a scarcity of prospectively collected data within most cultures, which limits the possibility to draw valid conclusions about causality. On the other hand, prospective studies might "lock" the data collection to measurement techniques that become obsolete during the observation period. In addition, new statistical methods may be needed to account for sequences of events and the longitudinal nature of late and long-term effects.

An equally or perhaps more important aspect pertains to the time span in question. Due to the late onset of adverse events, information that becomes available is in many instances the consequences of yesterday's treatment regimens, as advances take place and protocols are updated regularly. The pace may, however, vary across cultures, which may further hamper comparative research efforts. The information obtained may be outdated, although relevant to the longer-term survivors exposed in the past. New treatments are often in part based on chemical compounds used earlier, and although not identical perhaps similar enough that conclusions may be drawn nonetheless. This has resulted in an increased focus on the potential role of intermediate outcomes. With rapid improvements in diagnostic procedures and treatment regimens, researchers must find a way to develop and utilize intermediate outcomes more frequently. Sophisticated mathematical modeling can predict outcomes before they actually occur, and thus allow for appropriate interventions in advance [57]. Intermediate outcomes may also help in determining the relative importance of different treatment regimens versus earlier diagnosis, helping to determine the value of screening. Cancer survivorship research thus benefits from multiple measurement techniques and designs because various research methods have the potential for providing diverse insights, within as well as across cultures.

\subsection{Challenge III: Avoiding Selection Bias in Initial Partici-} pation and Followup. Previous studies in the field of cancer survivorship have by and large utilized information provided by affected individuals through interviews or questionnaires. Unfortunately, response rates have been relatively low and not representative of the entire population of survivors $[1,58]$. Participation and response rates are in general related to many of the exposures and outcomes of interest in cancer survivorship research, and are likely to differ within and across cultures $[27,28,58]$. In studies based on membership in groups, organizations, or catchment areas, further selection bias may be present [59]. Surprisingly, not all cancer survivors are aware of or accurately report their diagnosis when asked specifically to do so $[60,61]$. These individuals are unlikely to participate in studies that are not registry-based and tend to have different characteristics than those who report their diagnosis [60]. Furthermore, similar baseline characteristics among responders and nonresponders have been shown to be insufficient evidence of nonresponse bias, as the two groups experience different long-term outcome rates [62]. In general, participation rates are on the decline today, across studies and in most societies [27,63]. With high social mobility in many western countries, many survivors are lost to followup.

The way research is conducted may create its own barriers. Recent administrative and legal changes across countries have required studies to have complicated informed consent [63]. This may increase the potential for bias, as a more complex consent process may make it more likely for healthier, more resourceful persons to participate [63, 64]. As study aims and measures become more sophisticated, more precise answers and more tests may be required from subjects. This can be time and energy consuming, and persons in poorer health or those experiencing adverse outcomes may be less likely to have the ability to participate [27, 64]. Sensitive topics are, in addition, more likely to invoke differential nonresponse rates, for instance sexual preferences and/or behavior [65]. Cultural norms and expectations may influence both response rates and the information subjects choose to share. Any or all of the factors listed above limit our ability to capture complete cohorts. This is unfortunate, as although the detailed tests, examinations, questionnaires, and/or interviews may reduce participation, they may at the same time increase construct validity for those participating. Achieving a more optimal balance between the two within and across cultures is thus a challenge.

\section{Discussion}

Cross-cultural comparisons are increasingly important to maximize the cost-benefit of conducting research without duplication of effort in cancer survivorship research. These 
comparisons will also help to determine the relative importance of cultural and societal structures. Although many consequences of cancer may be culturally dependent, it is likely that research questions and results may be transferred to cultures with similar populations, cancer burdens, health systems, and welfare structures. How to interpret findings for populations that differ substantially in any of these respects remains unclear, and culture-specific research in these areas remain warranted.

Cancer survivor research encompasses the fields of clinical, behavioral, sociologic, anthropologic, economic, and health services research. We need an increased focus on genuine interdisciplinary research efforts, where persons from different backgrounds work together in teams to solve problems, thus coalescing their unique and shared experiences to create new theoretical and conceptual models.

The longitudinal nature of cancer survivorship research remains challenging, as cultures' rate of uptake of new detection methods, treatments, as well as research ideas and methods differ. Current findings may reflect earlier diagnostic criteria and treatment options and not necessarily improvements in treatment or "real" changes in other areas. Methods to simultaneously incorporate aspects of survivorship at individual, group, and societal levels, separately as well as jointly, and changes that occur within and across these levels over time are needed.

As populations grow and age so will the number of cancer survivors, requiring an increased emphasis on optimal resource management. Reasonable health care costs, adequate personnel and services, and equal access to care will be important for both the individual cancer patient and the greater community. It is thus pertinent that the current focus on individuals, directly or indirectly affected, shifts to also include effects at societal levels. In this effort, multilevel studies will become increasingly relevant.

\section{Suggested Future Courses of Action}

We recommend that whenever possible, data should be made comparable across studies, cultures, regions, and systems to facilitate larger and more diverse studies and valid results in universal and specific contexts. To facilitate this, cancer registries can expand to include agreed upon factors important for survivorship as a foundation for prospective research, nationally and cross-nationally. Further, analytic tools must be identified or developed that will enable us to capture needs of survivors, families, caregivers, clinicians, health and welfare policy makers and enforcers and alsotaking this a step further-make findings at group levels relevant at an individual level for those affected and those who provide care.

To address the broad aspects of survivorship we need data, methods, and study designs that can be used to compare across cultures. Survivorship registries, currently being developed in Norway, and in Vermont, USA, and likely other places, will yield important information on the psychosocial needs and resources, use of diagnostic tests, treatments received, and risks of recurrences and morbidities, along with other relevant long-term outcomes and thus provide a knowledge base for further survivorship research. In Vermont over 2000 adult cancer survivors representing nine different cancers are available to be invited into research studies [58]. Basic demographic and cancer information is known for all the participants. In Norway, national survivorship registries for prostate and colorectal cancer which contain information on both initial and recurrent cancer and treatment have been established at the Cancer Registry of Norway. These registries may be linked to several other national registries to obtain for instance cause of death, medication use, income, education, employment and marital status. They can also be used to identify patients to conduct more in depth studies, for example, whether persons' quality of life after rectal cancer depend on whether or not they have a stoma [66].

\section{Conclusion}

Research hypotheses, questions, and findings that generalize to other larger or more diverse populations can be identified to increase the rate of new findings. To optimize data collection we must strive to increase the comparability across studies, cultures, regions, and systems to enhance validity in universal and specific contexts and thus achieve power to make valid conclusions also on negative results. The needs of survivors, families/caregivers, clinicians, health and welfare policy makers and enforcers can be identified and addressed in cross-cultural comparative research. New study designs and statistical methods will enhance all aspects of cancer survivorship research. Prioritizing the expansion of cancer registries to include survivorship data elements will facilitate prospective research, nationally and cross-culturally. The relative importance of research that benefits individuals versus the larger society needs to be assessed, and findings at group levels can be made relevant at an individual level for those affected and those aiming to assist. Our future lies with interdisciplinary and cross-cultural survivorship research.

In conclusion, we hope that a comparative perspective will be considered when new survivorship cohorts are established and in the planning and implementation of new survivorship studies. In cancer survivorship care, research-based knowledge should be applied. Practice must reciprocally influence research. In a broader perspective, cross-cultural comparisons may translate to health and welfare policy through health services research on cancer survivorship.

\section{Conflict of interests}

No conflict of interests exists.

\section{Acknowledgment}

This research was supported by a grant from the Norwegian Research Council. The authors contributed equally to this work. 


\section{References}

[1] M. Hewitt, E. Stovall, and S. Greenfield, From Cancer Patient to Cancer Survivor: Lost in Transition, National Academies Press, Washington, DC, USA, 2005.

[2] J. H. Rowland and K. M. Bellizzi, "Cancer survivors and survivorship research: a reflection on today's successes and tomorrow's challenges," Hematology/Oncology Clinics of North America, vol. 22, no. 2, pp. 181-200, 2008.

[3] J. H. Rowland, "Cancer survivorship: rethinking the cancer control continuum," Seminars in Oncology Nursing, vol. 24, no. 3, pp. 145-152, 2008.

[4] M. Feuerstein, "Defining cancer survivorship," Journal of Cancer Survivorship, vol. 1, no. 1, pp. 5-7, 2007.

[5] Office of Cancer Survivorship. National Cancer Insitute, http://dccps.nci.nih.gov/ocs/.

[6] P. A. Ganz, "Why and how to study the fate of cancer survivors: observations from the clinic and the research laboratory," European Journal of Cancer, vol. 39, no. 15, pp. 2136-2141, 2003.

[7] M. M. Hawkins, E. R. Lancashire, D. L. Winter et al., "The British Childhood Cancer Survivor Study: objectives, methods, population structure, response rates and initial descriptive information," Pediatric Blood and Cancer, vol. 50, no. 5, pp. 1018-1025, 2008.

[8] M. L. McBride, P. C. Rogers, S. B. Sheps et al., "Childhood, adolescent, and young adult cancer survivors research program of British Columbia: objectives, study design, and cohort characteristics," Pediatric Blood and Cancer, vol. 55, no. 2, pp. 324-330, 2010.

[9] K. Yamaguchi, "Overview of cancer control programs in Japan," Japanese Journal of Clinical Oncology, vol. 32, pp. S22S31, 2002.

[10] E. G. Eakin, D. R. Youlden, P. D. Baade et al., "Health status of long-term cancer survivors: results from an Australian population-based sample," Cancer Epidemiology Biomarkers and Prevention, vol. 15, no. 10, pp. 1969-1976, 2006.

[11] A. Syse, Ø. Kravdal, and S. Tretli, "Parenthood after cancer-a population-based study," Psychooncology, vol. 16, no. 10, pp. 920-927, 2007.

[12] M. Dorval, S. Guay, M. Mondor et al., "Couples who get closer after breast cancer: frequency and predictors in a prospective investigation," Journal of Clinical Oncology, vol. 23, no. 15, pp. 3588-3596, 2005.

[13] K. Y. Yoo, "Cancer control activities in the Republic of Korea," Japanese Journal of Clinical Oncology, vol. 38, no. 5, pp. 327333, 2008.

[14] V. Mattioli, R. Montanaro, and F. Romito, "The Italian response to cancer survivorship research and practice: developing an evidence base for reform," Journal of Cancer Survivorship, vol. 4, no. 3, pp. 284-289, 2010.

[15] E. Maunsell, M. Drolet, J. Brisson, C. Brisson, B. Mâsse, and L. Deschênes, "Work situation after breast cancer: results from a population-based study," Journal of the National Cancer Institute, vol. 96, no. 24, pp. 1813-1822, 2004.

[16] F. Joly, M. Henry-Amar, P. Arveux et al., "Late psychosocial sequelae in Hodgkin's disease survivors: a French populationbased case-control study," Journal of Clinical Oncology, vol. 14, no. 9, pp. 2444-2453, 1996.

[17] M. Schroevers, A. V. Ranchor, and R. Sanderman, "Adjustment to cancer in the 8 years following diagnosis: a longitudinal study comparing cancer survivors with healthy individuals," Social Science and Medicine, vol. 63, no. 3, pp. 598-610, 2006.
[18] E. R. Spelten, J. H. A. M. Verbeek, A. L. J. Uitterhoeve et al., "Cancer, fatigue and the return of patients to work-a prospective cohort study," European Journal of Cancer, vol. 39, no. 11, pp. 1562-1567, 2003.

[19] K. Carlsen, S. O. Dalton, K. Frederiksen, F. Diderichsen, and C. Johansen, "Are cancer survivors at an increased risk for divorce? A Danish cohort study," European Journal of Cancer, vol. 43, no. 14, pp. 2093-2099, 2007.

[20] S. Kumar, A. J. Shaikh, S. Khalid, and N. Masood, "Influence of patient's perceptions, beliefs and knowledge about cancer on treatment decision making in Pakistan," Asian Pacific Journal of Cancer Prevention, vol. 11, no. 1, pp. 251-255, 2010.

[21] "World cancer report 2008," http://www.iarc.fr/en/publications/pdfs-online/wcr/2008/wcr_2008.pdf.

[22] GLOBOCAN, "Cancer incidence and mortality worldwide," 2008, http://globocan.iarc.fr/.

[23] A. Jemal, M. M. Center, C. DeSantis, and E. M. Ward, "Global patterns of cancer incidence and mortality rates and trends," Cancer Epidemiology Biomarkers and Prevention, vol. 19, no. 8, pp. 1893-1907, 2010.

[24] K. M. Bellizzi, "Expressions of generativity and posttraumatic growth in adult cancer survivors," International Journal of Aging and Human Development, vol. 58, no. 4, pp. 267-287, 2004.

[25] A. Syse and O. Kravdal, "Does cancer affect the divorce rate?" Demographic Research, vol. 16, article 15, pp. 469-492, 2007.

[26] L. B. Travis, S. D. Fosså, S. J. Schonfeld et al., "Second cancers among 40,576 testicular cancer patients: focus on long-term survivors," Journal of the National Cancer Institute, vol. 97, no. 18, pp. 1354-1365, 2005.

[27] C. R. Baquet, G. L. Ellison, and S. I. Mishra, "Analysis of Maryland cancer patient participation in National Cancer Institute-supported cancer treatment clinical trials," Journal of Health Care for the Poor and Underserved, vol. 20, no. 2, pp. 120-134, 2009.

[28] S. R. Sears, A. L. Stanton, L. Kwan et al., "Recruitment and retention challenges in breast cancer survivorship research: results from a multisite, randomized intervention trial in women with early stage breast cancer," Cancer Epidemiology Biomarkers and Prevention, vol. 12, no. 10, pp. 1087-1090, 2003.

[29] L. Thorsen, E. Skovlund, S. B. Strømme, K. Hornslien, A. A. Dahl, and S. D. Fosså, "Effectiveness of physical activity on cardiorespiratory fitness and health-related quality of life in young and middle-aged cancer patients shortly after chemotherapy," Journal of Clinical Oncology, vol. 23, no. 10, pp. 2378-2388, 2005.

[30] J. Vardy, S. Rourke, and I. F. Tannock, "Evaluation of cognitive function associated with chemotherapy: a review of published studies and recommendations for future research," Journal of Clinical Oncology, vol. 25, no. 17, pp. 2455-2463, 2007.

[31] M. L. Irwin, E. J. Aiello, A. McTiernan et al., "Physical activity, body mass index, and mammographic density in postmenopausal breast cancer survivors," Journal of Clinical Oncology, vol. 25, no. 9, pp. 1061-1066, 2007.

[32] K. D. Stein, K. L. Syrjala, and M. A. Andrykowski, "Physical and psychological long-term and late effects of cancer," Cancer, vol. 112, no. 11, pp. 2577-2592, 2008.

[33] I. F. Tannock, T. A. Ahles, P. A. Ganz, and F. S. Van Dam, "Cognitive impairment associated with chemotherapy for cancer: report of a workshop," Journal of Clinical Oncology, vol. 22, no. 11, pp. 2233-2239, 2004. 
[34] J. A. Paice, "Assessment of symptom clusters in people with cancer," Journal of the National Cancer Institute. Monographs, no. 32, pp. 98-102, 2004.

[35] M. L. Lindbohm, E. Kuosma, T. Taskila et al., "Cancer as the cause of changes in work situation (a NOCWO study)," Psychooncology, vol. 20, no. 8, pp. 805-812, 2010.

[36] J. F. Steiner, T. A. Cavender, D. S. Main, and C. J. Bradley, "Assessing the impact of cancer on work outcomes: what are the research needs?" Cancer, vol. 101, no. 8, pp. 1703-1711, 2004.

[37] W. Demark-Wahnefried, N. M. Aziz, J. H. Rowland, and B. M. Pinto, "Riding the crest of the teachable moment: promoting long-term health after the diagnosis of cancer," Journal of Clinical Oncology, vol. 23, no. 24, pp. 5814-5830, 2005.

[38] A. A. Thornton and M. A. Perez, "Posttraumatic growth in prostate cancer survivors and their partners," Psychooncology, vol. 15, no. 4, pp. 285-296, 2006.

[39] Y. Kim and B. A. Given, "Quality of life of family caregivers of cancer survivors: across the trajectory of the illness," Cancer, vol. 112, no. 11, pp. 2556-2568, 2008.

[40] N. M. Aziz and J. H. Rowland, "Cancer survivorship research among ethnic minority and medically underserved groups," Oncology Nursing Forum, vol. 29, no. 5, pp. 789-801, 2002.

[41] T. Smith, K. D. Stein, C. C. Mehta et al., "The rationale, design, and implementation of the American Cancer Society's studies of cancer survivors," Cancer, vol. 109, no. 1, pp. 1-12, 2007.

[42] S. Shahid, L. Finn, D. Bessarab et al., "Nowhere to room ... nobody told them': logistical and cultural impediments to Aboriginal peoples' participation in cancer treatment," Australian Health Review, vol. 35, no. 2, pp. 235-241, 2011.

[43] C. H. Chen, S. T. Tang, and C. H. Chen, "Meta-analysis of cultural differences in Western and Asian patient-perceived barriers to managing cancer pain," Palliative Medicine. In press.

[44] National Cancer Institute, "Multilevel interventions in cancer control," http://cancercontrol.cancer.gov/mli/documents.htm.

[45] L. M. McGregor, M. L. Metzger, R. Sanders, and V. M. Santana, "Pediatric cancers in the new millennium: dramatic progress, new challenges," Oncology, vol. 21, no. 7, pp. 809-820, 2007.

[46] L. W. Lund, K. Schmiegelow, C. Rechnitzer et al., "A systematic review of studies on psychosocial late effects of childhood cancer: structures of society and methodological pitfalls may challenge the conclusions," Pediatric Blood \& Cancer, vol. 56, no. 4, pp. 532-543, 2011.

[47] P. A. Ganz, A. Coscarelli, C. Fred, B. Kahn, M. L. Polinsky, and L. Petersen, "Breast cancer survivors: psychosocial concerns and quality of life," Breast Cancer Research and Treatment, vol. 38, no. 2, pp. 183-199, 1996.

[48] A. Syse, S. Tretli, and Ø. Kravdal, "Cancer's impact on employment and earnings-a population-based study from Norway," Journal of Cancer Survivorship, vol. 2, no. 3, pp. 149158, 2008.

[49] C. J. Bradley, D. Neumark, Z. Luo, and M. Schenk, "Employment and cancer: findings from a longitudinal study of breast and prostate cancer survivors," Cancer Investigation, vol. 25, no. 1, pp. 47-54, 2007.

[50] J.S. Banthin and D. M. Bernard, "Changes in financial burdens for health care: national estimates for the population younger than 65 years, 1996 to 2003," Journal of the American Medical Association, vol. 296, no. 22, pp. 2712-2719, 2006.

[51] C. J. Bradley, D. Neumark, Z. Luo, and H. L. Bednarek, "Employment-contingent health insurance, illness, and labor supply of women: evidence from married women with breast cancer," Health Economics, vol. 16, no. 7, pp. 719-737, 2007.

[52] S. B. Gudbergsson, S. D. Fosså, P. A. Ganz, B. J. Zebrack, and A. A. Dahl, "The associations between living conditions, demography, and the "impact of cancer" scale in tumor-free cancer survivors: a NOCWO study," Supportive Care in Cancer, vol. 15, no. 11, pp. 1309-1318, 2007.

[53] N. J. M. Pearce, R. Sanson-Fisher, and H. S. Campbell, "Measuring quality of life in cancer survivors: a methodological review of existing scales," Psychooncology, vol. 17, no. 7, pp. 629-640, 2008.

[54] H. S. Campbell, R. Sanson-Fisher, D. Turner, L. Hayward, X. S. Wang, and J. Taylor-Brown, "Psychometric properties of cancer survivors' unmet needs survey," Supportive Care in Cancer, pp. 1-10, 2010.

[55] C. M. Crespi, P. A. Ganz, L. Petersen, A. Castillo, and B. Caan, "Refinement and psychometric evaluation of the impact of cancer scale," Journal of the National Cancer Institute, vol. 100, no. 21, pp. 1530-1541, 2008.

[56] S. J. Nechuta, B. J. Caan, W. Y. Chen et al., "The after breast cancer pooling project: rationale, methodology, and breast cancer survivor characteristics," Cancer Causes Control, vol. 22, no. 9, pp. 1319-1331, 2011.

[57] K. M. Kuntz, I. Lansdorp-Vogelaar, C.M. Rutter et al., "A systematic comparison of microsimulation models of colorectal cancer: the role of assumptions about adenoma progression," Medical Decision Making, vol. 31, no. 4, pp. 530-539, 2011.

[58] B. M. Geller, J. Mace, P. Vacek, A. Johnson, C. Lamer, and D. Cranmer, "Are cancer survivors willing to participate in research?" Journal of Community Health, vol. 36, no. 5, pp. 772-778, 2011.

[59] D. Katz, T. M. Koppie, D. Wu et al., "Sociodemographic characteristics and health related quality of life in men attending prostate cancer support groups," Journal of Urology, vol. 168, no. 5, pp. 2092-2096, 2002.

[60] C. Nord, A. Mykletun, and S. D. Fosså, "Cancer patients' awareness about their diagnosis: a population-based study," Journal of Public Health Medicine, vol. 25, no. 4, pp. 313-317, 2003.

[61] L. Abraham, B. M. Geller, B. C. Yankaskas et al., "Accuracy of self-reported breast cancer among women undergoing mammography," Breast Cancer Research and Treatment, vol. 118, no. 3, pp. 583-592, 2009.

[62] J. Vestbo and F. V. Rasmussen, "Baseline characteristics are not sufficient indicators of non-response bias follow up studies," Journal of Epidemiology and Community Health, vol. 46, no. 6, pp. 617-619, 1992.

[63] O. Berger, B. H. Grønberg, K. Sand, S. Kaasa, and J. H. Loge, "The length of consent documents in oncological trials is doubled in twenty years," Annals of Oncology, vol. 20, no. 2, pp. 379-385, 2009.

[64] J. H. Stewart, A. G. Bertoni, J. L. Staten, E. A. Levine, and C. P. Gross, "Participation in surgical oncology clinical trials: gender-, race/ethnicity-, and age-based disparities," Annals of Surgical Oncology, vol. 14, no. 12, pp. 3328-3334, 2007.

[65] R. Jenkins, L. R. Schover, R. T. Fouladi et al., "Sexuality and health-related quality of life after prostate cancer in AfricanAmerican and white men treated for localized disease," Journal of Sex and Marital Therapy, vol. 30, no. 2, pp. 79-93, 2004.

[66] M. G. Guren, M. T. Eriksen, J. N. Wiig et al., "Quality of life and functional outcome following anterior or abdominoperineal resection for rectal cancer," European Journal of Surgical Oncology, vol. 31, no. 7, pp. 735-742, 2005. 


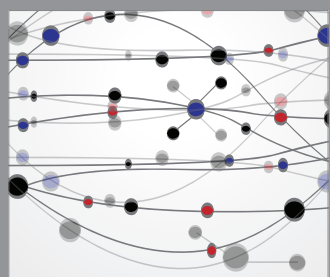

The Scientific World Journal
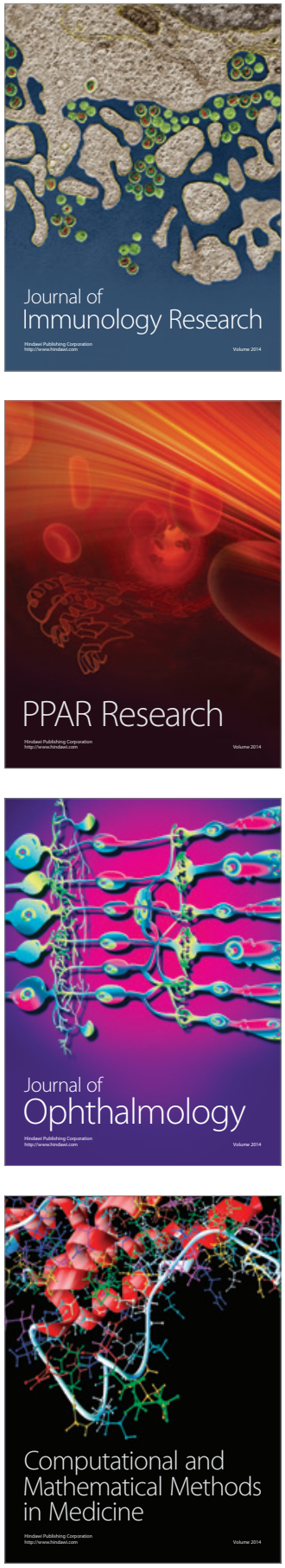

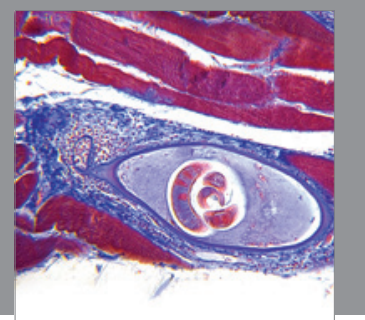

Gastroenterology

Research and Practice
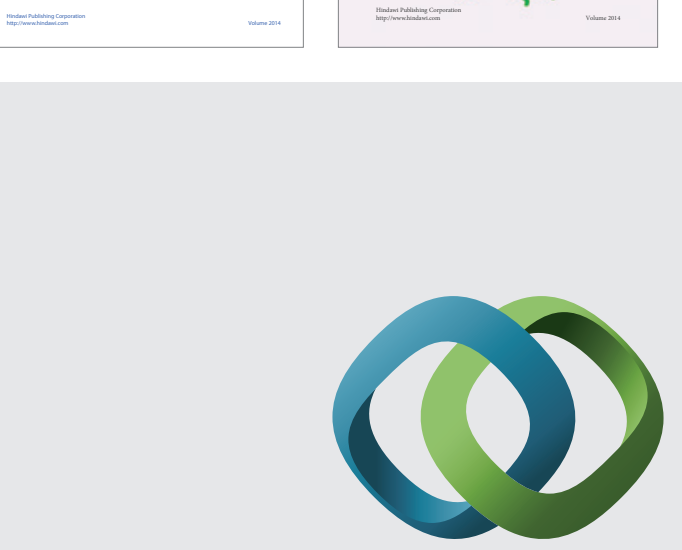

\section{Hindawi}

Submit your manuscripts at

http://www.hindawi.com
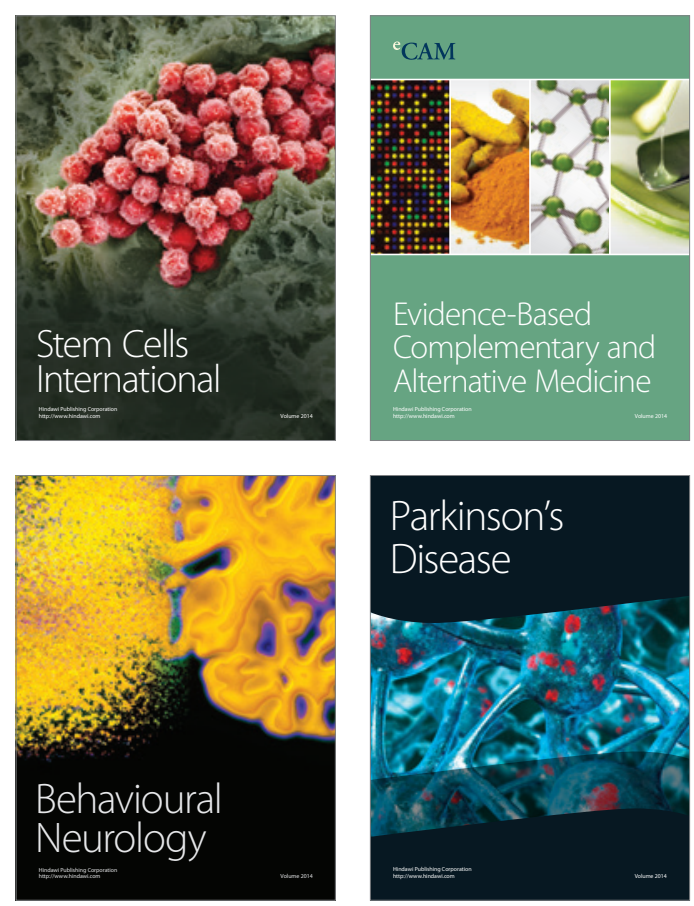

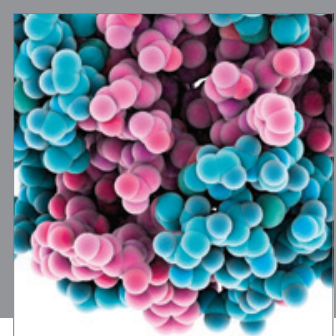

Journal of
Diabetes Research

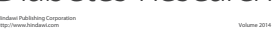

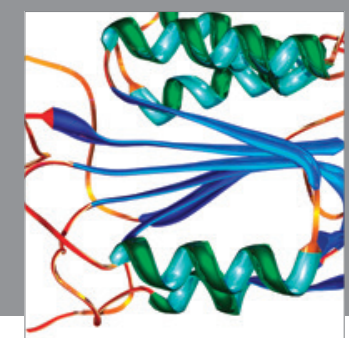

Disease Markers
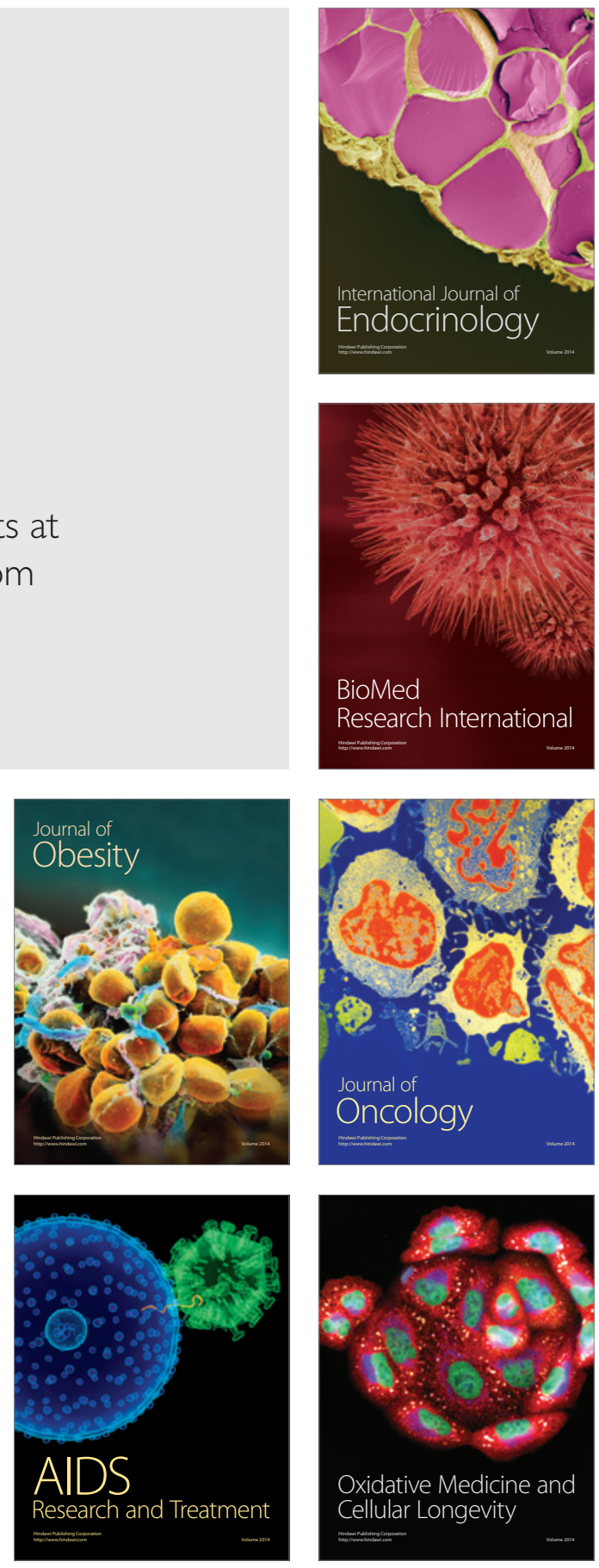